\title{
Les conditions de travail des salariés dans l'Union européenne à quinze selon les formes d'organisation
}

Working conditions of EU-15 workers according to the forms of work organisation

\section{Antoine Valeyre}

\section{OpenEdition}

Journals

Édition électronique

URL : http://journals.openedition.org/travailemploi/2185

DOI : 10.4000/travailemploi.2185

ISSN : $1775-416 \mathrm{X}$

Éditeur

DARES - Ministère du Travail

\section{Édition imprimée}

Date de publication : 30 décembre 2007

Pagination : $35-47$

ISSN : 0224-4365

Référence électronique

Antoine Valeyre, « Les conditions de travail des salariés dans l'Union européenne à quinze selon les formes d'organisation », Travail et Emploi [En ligne], 112 | octobre-décembre 2007, mis en ligne le 05 novembre 2010, consulté le 10 décembre 2020. URL : http://journals.openedition.org/travailemploi/ 2185 ; DOI : https://doi.org/10.4000/travailemploi.2185 


\title{
Les conditions de travail des salariés dans I'Union européenne à quinze selon les formes d'organisation
}

\author{
Antoine Valeyre $(*)$
}

L'article analyse les conditions de travail dans les pays de l'Union européenne à quinze à partir de la troisième enquête européenne sur les conditions de travail réalisée en mars 2000. Il s'interroge sur les effets de quatre modèles d'organisation du travail: les modèles d'organisation apprenante, en lean production, taylorienne et simple, et passe en revue tout à tour les contraintes physiques, les horaires et les cadences de travail. Il conclut que l'organisation apprenante est, sur la plupart des critères, l'organisation associée aux meilleures conditions de travail. Mais les innovations organisationnelles depuis la fin des années 1980 intègrent rarement une tentative d'amélioration des conditions de travail et la question garde toute son actualité.

Face aux limites, tant économiques que sociales, rencontrées par les organisations tayloriennes/ fordiennes du travail, de multiples innovations organisationnelles ont été mises en œuvre depuis une trentaine d'années. Elles visent à améliorer les performances économiques et productives des entreprises confrontées à un environnement de marché plus instable et plus diversifié, à une concurrence exacerbée et mondialisée et à une demande plus exigeante en termes de variété, de qualité, de délais, de réactivité et de vitesse de renouvellement des produits. Elles visent également à prendre en compte 1'aspiration à plus d'autonomie et d'initiative dans le travail qu'expriment de nombreux salariés dans un contexte d'élévation des niveaux de formation et, tout au moins dans les années 1970, à répondre à un rejet croissant des conditions de travail associées aux organisations tayloriennes/fordiennes du travail, qui se manifeste par une montée de l'absentéisme, du turnover et des conflits sociaux.

Les innovations organisationnelles qui tentent de dépasser les obstacles économiques et sociaux auxquels se heurte le modèle taylorien/fordien conduisent à des transformations profondes des pratiques de travail. Des expériences d'élargissement et d'enrichissement des tâches et de groupes semi-autonomes de production inspirées du modèle sociotechnique du Tavistock Institute (EMERY, TRIST, 1960) ont été réalisées dès les années 1970 par des entreprises pionnières, notamment dans les pays

(*) Centre d'études de l'emploi-CNRS, antoine.valeyre@, laposte.net. Cet article s'appuie sur les résultats d'une recherche financée par la DARES (cf. VALEYRE, 2006a et b). L'auteur tient à remercier Thomas Coutrot et Michel Gollac pour leurs remarques et suggestions sur des versions antérieures de ce texte. scandinaves, et de façon beaucoup plus limitée en France. Elles ont été relayées depuis les années 1980 par la diffusion, souvent concomitante, de multiples dispositifs organisationnels tels que le travail en équipes (équipes autonomes(1), équipes transversales et pluridisciplinaires comme les groupes de projets, les cercles de qualité ou les groupes de résolution de problèmes), la polyvalence, le raccourcissement des lignes hiérarchiques, les démarches de qualité totale et la production en flux tendus. Ces dispositifs relèvent des principes du modèle de la lean production qui est souvent considéré comme constitutif d'un nouveau modèle d'organisation du travail dont l'efficacité en ferait un nouveau one best way qui supplanterait le modèle taylorien/fordien (Womack, John, Roos, 1990; MacDuffie, KrafciK, 1992). Ils ont connu une diffusion rapide aux USA depuis le milieu des années 1980 (Osterman, 1994 et 2000), puis en Europe à partir de la fin des années 1980. Des enquêtes statistiques comme les enquêtes COI ou REPONSE montrent l'ampleur de cette diffusion en France dans les années 1990 (Askenazy, Caroli, 2003; Coutrot, 1995 et 2000; Greenan, Hamon-Cholet, 2000).

\footnotetext{
(1) Précisons que la notion d'équipe autonome de travail présente des contenus très divers. Ainsi, la conception des équipes autonomes de travail (teamwork) des organisations en lean production diffère profondément de celle des groupes semi autonomes de production du modèle sociotechnique scandinave. Dans ces derniers, une place bien plus grande est faite à l'auto-organisation. Le travail est librement organisé dans le groupe, les objectifs de production sont souvent négociés avec la hiérarchie et le porte-parole est choisi par le groupe, ce qui n'est pas le cas dans les équipes de la lean production (DuRAND, Stewart, Castillo, 1998; Fröhlich, Pekruhl, 1996) qui, en conséquence, se trouvent beaucoup moins propices au développement de l'autonomie au travail des salariés.
} 
Paradoxalement, les innovations organisationnelles des vingt dernières années ne s'accompagnent pas d'une amélioration des conditions de travail, alors même que des tendances lourdes comme la tertiarisation de l'économie ou l'automatisation et l'informatisation de la production constituent des facteurs structurels favorables à la réduction des pénibilités et des risques professionnels. Les enquêtes statistiques sur les conditions de travail ou sur les risques professionnels mettent plutôt en évidence une tendance à la dégradation de la situation. Ainsi, sur la base des trois premières enquêtes européennes sur les conditions de travail, on observe dans l'Union européenne, au cours des années 1990, une légère dégradation de nombreuses conditions physiques de travail, comme l'exposition à des postures douloureuses ou fatigantes, au port de charges lourdes, au bruit ou à la chaleur, que ne compensent pas la légère amélioration concernant les expositions au froid ou à l'inhalation des fumées et vapeurs dangereuses (Merllié, Paoli, 2001; Daubas-Letourneux, Thébaud-Mony, 2003). Simultanément, et de façon liée, le travail tend à s'intensifier sous la pression de cadences élevées ou de délais serrés. En France, sur la période de 1984 à 1998, les enquêtes «Conditions de travail» conduisent à des constats analogues en matière de conditions physiques de travail et d'intensification du travail (Askenazy 2004; Bué, Coutrot, Puech, 2004). Sur la période plus récente des dix dernières années, l'enquête SUMER de 2003 montre également une tendance à l'accroissement de nombreux risques et pénibilités physiques du travail (ARNAUDO et al., 2004).

Dans un ouvrage récent, AsKenAzY (2004) explique ce paradoxe par les dynamiques d'intensification du travail et de cumul des contraintes de rythme qui accompagnent les pratiques de travail innovantes et simultanément contribuent à détériorer les conditions de travail. Ces dynamiques s'observent aussi bien en France (CARTRON, Gollac, 2006; Gollac, Volkoff, 1996; Valeyre, 2004) qu'au niveau européen (BoISARD et al., 2002) et jusqu'au milieu des années 1990 aux USA (AskeNAZY, 2002, 2004). De fait, les contraintes temporelles imposées par la tension des flux, par les exigences de la demande et par les urgences dans le traitement des incidents et des dysfonctionnements de la production, réduisent les marges de manœuvre dont disposent les salariés pour construire de bons compromis entre les contraintes de la production et la préservation de la qualité de leurs conditions de travail, alors même qu'une certaine autonomie procédurale leur est octroyée. Elles rendent l'exposition aux pénibilités et aux risques professionnels plus difficiles à éviter, par exemple faute de temps pour choisir les gestes et les postures les mieux adaptés à des contraintes physiques à risque ou pour prendre les précautions qu'impose un travail en contact avec des nuisances physicochimiques (DAVEZIES, 1999; Gollac, VolKoff, 2000).
Cependant, les pratiques innovantes de travail sur lesquelles se fondent cette analyse du paradoxe relèvent essentiellement du modèle de la lean production dans la mesure où sont privilégiés des dispositifs organisationnels comme la rotation des tâches, le travail en équipes, la production en juste à temps ou la gestion de la qualité totale. La question se pose donc de savoir si les innovations organisationnelles ne se diffusent pas aussi dans d'autres formes d'organisation du travail plus respectueuses de la qualité de vie au travail et plus propices à l'amélioration des conditions de travail, dans le prolongement des principes du modèle sociotechnique scandinave. Dans ce cas, le paradoxe ne concernerait qu'une partie des organisations innovantes et les voies de son dépassement pourraient s'appuyer sur des pratiques s'inspirant d'autres modèles que celui de la lean production. De fait, la thèse de l'unicité des nouveaux modèles d'organisation du travail inspirés des principes de la lean production ne fait pas l'unanimité. À la thèse du nouveau one best way organisationnel lié à la lean production s'oppose celle de la pluralité des nouveaux modèles d'organisation du travail. Dans le contexte américain, Appelbaum et Batt (1994) identifient deux modèles efficients, celui de la lean production et celui de la «production en équipes autonomes à l'américaine» qui combine les principes du modèle sociotechnique suédois et ceux du management de la qualité totale. Coutrot (1998) distingue également deux modèles d'organisation innovants dans les entreprises françaises, l'un relevant du post-fordisme et l'autre du néofordisme de l'autonomie contrôlée. Les travaux de Boyer et Freyssenet (2000) portant sur l'industrie automobile à échelle mondiale contribuent aussi à alimenter la thèse de la diversité des nouveaux modèles productifs. Un travail récent fondé sur les données de l'enquête européenne sur les conditions de travail de 2000 montre que dans l'Union européenne des quinze les formes d'organisation du travail liées à la diffusion des innovations organisationnelles ne relèvent pas d'un modèle unique, mais de deux: le modèle relativement décentralisé des formes d'organisation apprenantes où les salariés disposent d'une large autonomie procédurale et ne subissent que de faibles contraintes temporelles dans leur travail, auquel se rattache le modèle sociotechnique scandinave; et le modèle plus hiérarchique des formes d'organisation en lean production où les salariés sont confrontés à des situations d'autonomie restreinte et contrôlée (LoRENZ, VALEYRe, 2005a). $\mathrm{Ce}$ résultat conduit à formuler l'hypothèse que les conditions de travail diffèrent de façon importante entre ces deux nouvelles formes d'organisation du travail. Plus précisément, les salariés bénéficieraient de meilleures conditions de travail dans le cadre des organisations apprenantes que dans celui des organisations en lean production, dans la mesure où ils disposent de marges plus importantes d'autonomie et d'initiative dans le travail et de moindres pressions temporelles, ce qui leur permet de mieux 
réguler leur activité de travail. Une hypothèse complémentaire est que les organisations apprenantes offriraient de meilleures conditions de travail que les organisations tayloriennes, contrairement aux organisations en lean production comme il a été constaté précédemment. La relation attendue entre innovations organisationnelles et meilleures conditions de travail s'observerait donc dans les organisations apprenantes, mais non dans les organisations en lean production. Le paradoxe des effets plutôt négatifs des innovations organisationnelles sur les conditions de travail ne vaudrait donc que pour les organisations en lean production.

Cet article se propose donc d'étudier les conditions de travail des salariés de l'Union européenne à quinze en fonction des formes d'organisation du travail. L'article présente tout d'abord le cadre méthodologique de la recherche et la typologie des formes d'organisation du travail - formes apprenantes, en lean production, tayloriennes et de structure simple - sur laquelle elle se fonde. Puis sont comparées les conditions de travail des salariés européens selon les différentes formes d'organisation du travail. Elles examinent successivement les conditions physiques de travail, les conditions horaires de travail et l'intensité du travail. Cet article met enfin évidence les différences de conditions de travail que l'on observe entre les deux formes innovantes d'organisation du travail, les formes apprenantes et les formes en lean production. Enfin, la conclusion propose quelques pistes d'interprétation de ces différences.

\section{Cadre méthodologique}

L'analyse comparative des conditions de travail selon les formes d'organisation du travail est fondée sur une exploitation statistique secondaire de l'enquête européenne sur les conditions de travail menée par la Fondation européenne pour l'amélioration des conditions de vie et de travail en 2000 ( $c f$. encadré 1). Comme l'étude s'intéresse aux pratiques organisationnelles mises en œuvre dans les entreprises, elle est focalisée sur les salariés des secteurs marchands, hors agriculture et services domestiques. En outre, les très petits établissements ne sont pas pris en compte car l'organisation du travail y est le plus souvent spécifique ou rudimentaire. Le champ de l'étude est donc constitué des salariés des établissements d'au moins dix personnes travaillant dans les secteurs marchands et non agricoles ou domestiques (ensemble des secteurs de la NACE à deux chiffres, à l'exclusion de l'administration publique et la sécurité sociale, l'éducation, la santé et l'action sociale, l'agriculture et la pêche, et les services domestiques). L'échantillon étudié compte 8081 individus représentatifs d'une population de près de 85 millions de salariés.

\section{Encadré 1}

\section{La troisième enquête européenne sur les conditions de travail}

La Fondation européenne pour l'amélioration des conditions de vie et de travail réalise périodiquement des enquêtes sur les conditions de travail. La troisième enquête a été effectuée dans les quinze pays membres de l'Union européenne en mars 2000. Environ 1500 personnes ayant un emploi ont été enquêtées dans chaque pays, à l'exception du Luxembourg avec environ 500 personnes. Au total, l'échantillon de l'enquête compte 21703 personnes.

L'enquête est fondée sur une méthode d'échantillonnage selon le principe des itinéraires aléatoires. La stratification est effectuée par régions, catégories urbaines, secteurs d'activité économique, groupes professionnels, genres et âges. Les entretiens ont été réalisés en face à face au domicile des personnes enquêtées.

Le questionnaire de l'enquête n'est pas exclusivement centré sur les conditions de travail. II comporte en effet huit volets thématiques: les caractéristiques structurelles des emplois (activité, profession...); l'environnement physique du travail; les temps et les horaires de travail; l'organisation du travail; les relations sociales dans le travail (communications, tensions...); la sécurité et la santé au travail; les situations familiales et les activités extraprofessionnelles; les rémunérations.

Les conditions de travail ne sont pas des variables qui s'imposent à l'observateur, mais des constructions sociales qui conduisent à détacher du travail certains de ses aspects, tout particulièrement lorsqu'ils soulèvent des problèmes de pénibilités ou d'atteintes à la santé (Gollac, VolKoff, 2000). Cette construction s'opère selon de multiples processus sociaux d'objectivation liés notamment aux progrès des connaissances scientifiques dans le domaine et à l'action des pouvoirs publics, des spécialistes de la santé et de la sécurité au travail, des organisations syndicales ou de groupes professionnels (Gollac, 1995). Les variables retenues dans l'analyse portent sur plusieurs aspects des conditions physiques de travail: les pénibilités physiques, les nuisances et les risques toxiques. Elles prennent aussi en compte le cadre temporel d'exercice de l'activité de travail sous deux angles, d'une part celui des conditions horaires du travail, dans leurs dimensions de durées de travail longues, d'horaires atypiques et d'horaires flexibles, et d'autre part celui des pressions temporelles dans le travail en termes d'intensité du travail. En revanche, la question de la charge mentale du travail (HAMON-CHOLET, 2004) n'est pas abordée, faute de données disponibles sur ce thème dans la source statistique utilisée.

Les formes d'organisation du travail sur lesquelles portent les comparaisons de conditions de travail se 
réfèrent à une typologie élaborée à partir des données de l'enquête européenne sur les conditions de travail de 2000 (Lorenz, Valeyre, 2005a). Cette typologie identifie quatre formes d'organisation du travail correspondant à des modèles couramment décrits dans la littérature organisationnelle, les formes apprenantes, en lean production, tayloriennes et de structure simple, dont les principales caractéristiques sont présentées dans l'encadré 2. Établie à partir de déclarations des salariés (2), elle est confortée par ses analogies avec les classifications des pratiques organisationnelles fondées sur des données d'établissements ou d'entreprises (Bunel et al., 2006; Coutrot, 1998; Moati, Pouquet, 2002). Elle met en évidence que les nouvelles formes d'organisation du travail se scindent en deux catégories bien distinctes, les organisations apprenantes en rupture avec le modèle taylorien et les organisations en lean production qui en conservent de nombreux traits (3). Elle montre également que les formes d'organisation tayloriennes ou de structure simple sont loin d'avoir disparu et continuent à s'imposer à une fraction importante de salariés de l'Union européenne.

La comparaison des variables de conditions de travail selon les formes d'organisation du travail est tout d'abord réalisée avec des méthodes de statistique descriptive. Cependant, il convient de prendre en compte le fait que les formes d'organisation du travail dépendent de caractéristiques structurelles liées à la nature des activités. Une étude réalisée sur la base des données de l'enquête européenne sur les conditions de travail de 2000 montre que les formes d'organisation du travail ont des disparités importantes selon les secteurs d'activité économique et la structure socioprofessionnelle de la main-d'œuvre (LOREnZ, VAleyre, 2005a) (4). Il n'y a toutefois pas de déterminisme structurel, comme on peut le constater avec la présence de chacune des quatre formes d'organisation du travail dans tous les secteurs d'activité. Si les organisations apprenantes sont prédominantes dans les secteurs des services et de l'énergie, regroupant notamment près de $60 \%$ des salariés des services aux entreprises, des activités financières et

(2) Construite sur la base de déclarations des salariés, cette typologie se propose d'identifier les formes d'organisation du travail à partir d'une classification des pratiques individuelles de travail. Elle comporte inévitablement certaines approximations taxonomiques, relativement limitées, comme le montrent les analyses structurelles par secteur, par profession ou par pays, réalisées sur les classes d'organisation du travail (LoRENZ, VALEYRE, 2005a). Une analyse menée au niveau des unités organisationnelles définies par des modes spécifiques de coordination du travail serait préférable. Mais aucune donnée n'est accessible à ce niveau, intermédiaire entre celui des entreprises ou de leurs établissements où coexistent fréquemment différentes formes d'organisation du travail et celui des postes de travail que permettent d'appréhender les enquêtes auprès des salariés.

(3) Sur les nouvelles formes d'organisation du travail, cf. notamment Boyer, DURAND (1993), LiNHART (1994); EDWARDS, GEARY, Sisson (2004).

(4) Pour une présentation plus détaillée des résultats de cette étude, $c f$. Lorenz, VAleyre (2004).
Encadré 2

\section{La typologie des formes d'organisation du travail}

La typologie des formes d'organisation du travail, construite à l'aide d'une méthode statistique de classification ascendante hiérarchique des salariés en fonction de quinze variables d'organisation, distingue quatre classes.

- La classe des organisations apprenantes regroupe des salariés qui disposent d'une forte autonomie dans le travail, autocontrôlent la qualité de leur travail et rencontrent fréquemment des situations d'apprentissage et de résolution de problèmes imprévus. Ils sont relativement nombreux à travailler en équipe. Ils exercent le plus souvent des tâches complexes, non monotones et non répétitives et subissent peu de contraintes de rythme. Cette classe s'apparente au modèle sociotechnique scandinave.

- La classe des organisations en lean production se caractérise par l'adoption de dispositifs organisationnels comme le travail en équipe, la rotation des tâches et la gestion de la qualité (autocontrôle de la qualité et respect de normes de qualité précises). Elle correspond typiquement au modèle de la lean production qui combine travail en groupe, polyvalence, qualité totale et flux tendus (MACDufFIE, KRAFCIK, 1992; Womack, Jones, Roos, 1990). Si, comme dans les organisations apprenantes, les salariés sont souvent confrontés à des situations d'apprentissage et de résolution de problèmes imprévus, ils bénéficient de bien moindres marges d'autonomie dans leur travail et sont soumis à de multiples contraintes de rythme. Ils rencontrent donc des situations de travail en «autonomie contrôlée » (APPAY, 1993; COUTROT, 1998).

- La classe des organisations tayloriennes, comme celle des organisations en lean production, associe des salariés astreints à d'importantes contraintes de rythme de travail, à des tâches répétitives et monotones, et à des normes de qualité précises. En revanche, leur travail présente une faible autonomie procédurale, un faible contenu cognitif et l'autocontrôle de la qualité est peu répandu. Cette classe relève du modèle taylorien d'organisation du travail, dans ses formes classiques, mais aussi dans ses formes assouplies en «taylorisme flexible» (BOYER, DURAND, 1993), comme le suggère la fréquence relative des pratiques de rotation des tâches.

- La classe des organisations de structure simple se caractérise par une faible fréquence du travail en équipe, de la rotation des tâches et de la gestion de la qualité. Le travail y est peu contraint dans ses rythmes et peu répétitif, mais relativement monotone et à faible contenu cognitif. Cette classe s'apparente au modèle des organisations de «structure simple» (MintZBERG, 1982) définies par une faible formalisation des procédures et un mode de contrôle par supervision directe exercée sur les salariés, soit par leur supérieur hiérarchique, soit par leur patron dans les petites entreprises. 
de la production et distribution d'électricité, gaz et eau, elles concernent néanmoins plus d'un salarié sur quatre dans presque tous les secteurs industriels. Si les organisations en lean production sont plus souvent adoptées dans les secteurs industriels, impliquant notamment deux salariés sur cinq dans la fabrication de matériels de transport, la fabrication électrique et électronique, les industries du bois, papier et carton, et l'édition et imprimerie, elles regroupent aussi plus d'un salarié sur six dans tous les secteurs de service, et même un sur quatre, soit une proportion proche de la moyenne, dans les transports, les postes et télécommunications, et l'hôtellerie et restauration. De même, si les organisations tayloriennes sont plus développées dans l'industrie, avec des proportions d'un quart des salariés ou plus dans les industries du textile, habillement et cuir, de l'agro-alimentaire, du bois, papier et carton, et de la fabrication de matériels de transport, elles touchent tous les secteurs de service et dépassent le niveau moyen avec un salarié sur six dans l'hôtellerie et restauration. En outre, à échelle européenne, il convient de prendre en compte la dimension nationale dans l'analyse, car les différences institutionnelles entre pays, notamment dans les modes de régulation des marchés et les systèmes de formation et de relations professionnelles, contribuent aussi à la différenciation des formes d'organisation du travail (Lorenz, Valeyre, 2005b). Par ailleurs, des facteurs comme les caractéristiques statutaires et démographiques de la main-d'œuvre jouent également dans les disparités de conditions de travail. En conséquence, afin de tenir compte de l'influence exercée par ces différents facteurs économiques, socioprofessionnels, démographiques ou nationaux, l'analyse comparative des conditions de travail en fonction des formes d'organisation du travail est complétée par des analyses de régression logistique effectuées avec des variables de contrôle structurel(5). Dans les modèles retenus, les variables expliquées sont les variables de conditions de travail et les variables explicatives sont d'une part les formes d'organisation du travail et d'autre part toute une série de variables de contrôle structurel: le secteur d'activité économique, la taille des établissements, le groupe socioprofessionnel, le pays, le type de contrat de travail, le genre, l'âge et l'ancienneté(6).

(5) Précisons que les résultats des régressions logistiques ne sont pas interprétés ici en termes de causalité comme pourrait y inviter le recours à des modèles économétriques. Ils sont simplement utilisés comme tests statistiques des relations entre formes d'organisation du travail et conditions de travail.

(6) La modalité de référence choisie pour la variable d'organisation du travail est constituée par les formes d'organisation tayloriennes. Les modalités de référence pour les variables de contrôle structurel sont: l'industrie pour les secteurs d'activité économique; la tranche de dix à quarante-neuf personnes pour la taille des établissements; les professions intermédiaires pour les groupes socioprofessionnels; l'Allemagne pour les pays; les CDI pour les types de contrats de travail; les hommes pour le genre; la tranche de 35 à 44 ans pour l'âge; et la tranche quatre à neuf ans pour l'ancienneté.

\section{Conditions physiques de travail}

L'enquête européenne sur les conditions de travail permet d'appréhender de nombreuses caractéristiques de conditions physiques de travail. Elles peuvent être distinguées en trois catégories: les pénibilités physiques liées aux conditions ergonomiques de travail (postures douloureuses ou fatigantes, manutentions de charges lourdes, mouvements répétitifs des mains ou des bras, vibrations mécaniques); les nuisances, notamment thermiques ou sonores, liées à l'environnement physique de travail (bruits intenses, chaleur, froid); et les risques toxiques liées à des expositions dangereuses (inhalations de substances dangereuses, contacts avec des substances dangereuses, irradiations). Ces différentes variables de conditions physiques de travail sont définies avec la spécification de durées d'exposition qui correspondent à des seuils couramment retenus pour traduire des facteurs de risque: au moins la moitié du temps de travail pour les variables de pénibilités physiques et de nuisances; au moins le quart du temps de travail pour les deux variables de risques toxiques relatives aux inhalations et aux contacts de substances dangereuses; et au moins un instant de travail pour la variable d'irradiations.

Les conditions physiques de travail varient considérablement en fonction des formes d'organisation du travail ( $c f$. tableau 1). Qu'il s'agisse de situations de pénibilités physiques, de nuisances sonores ou thermiques ou de risques toxiques, les salariés se trouvent beaucoup plus exposés dans les organisations en lean production et les organisations tayloriennes que dans les organisations apprenantes et les organisations de structure simple. En outre, les nuisances thermiques liées à la chaleur ou au froid et les risques toxiques d'inhalations ou de contacts dangereux sont nettement plus importants dans les organisations en lean production que dans les organisations tayloriennes. À l'inverse, c'est plutôt dans les organisations tayloriennes que l'exposition à des pénibilités physiques est la plus fréquente, très nettement en ce qui concerne les postures douloureuses ou fatigantes, les mouvements répétitifs des mains ou des bras et les vibrations mécaniques.

Le contrôle de l'influence de facteurs susceptibles de jouer dans la détermination des formes d'organisation du travail (activité économique, taille, groupe professionnel, pays, type de contrat de travail, genre, âge et ancienneté) à l'aide des régressions logistiques des variables de conditions physiques de travail en fonction des formes d'organisation du travail et de variables de contrôle structurel, comme il est proposé dans la présentation méthodologique, ne modifie pas fondamentalement les observations précédentes. On le vérifie clairement avec les odds ratios figurant 
Tableau 1

\section{Conditions physiques de travail selon les formes d'organisation du travail ( \% de salariés exposés)}

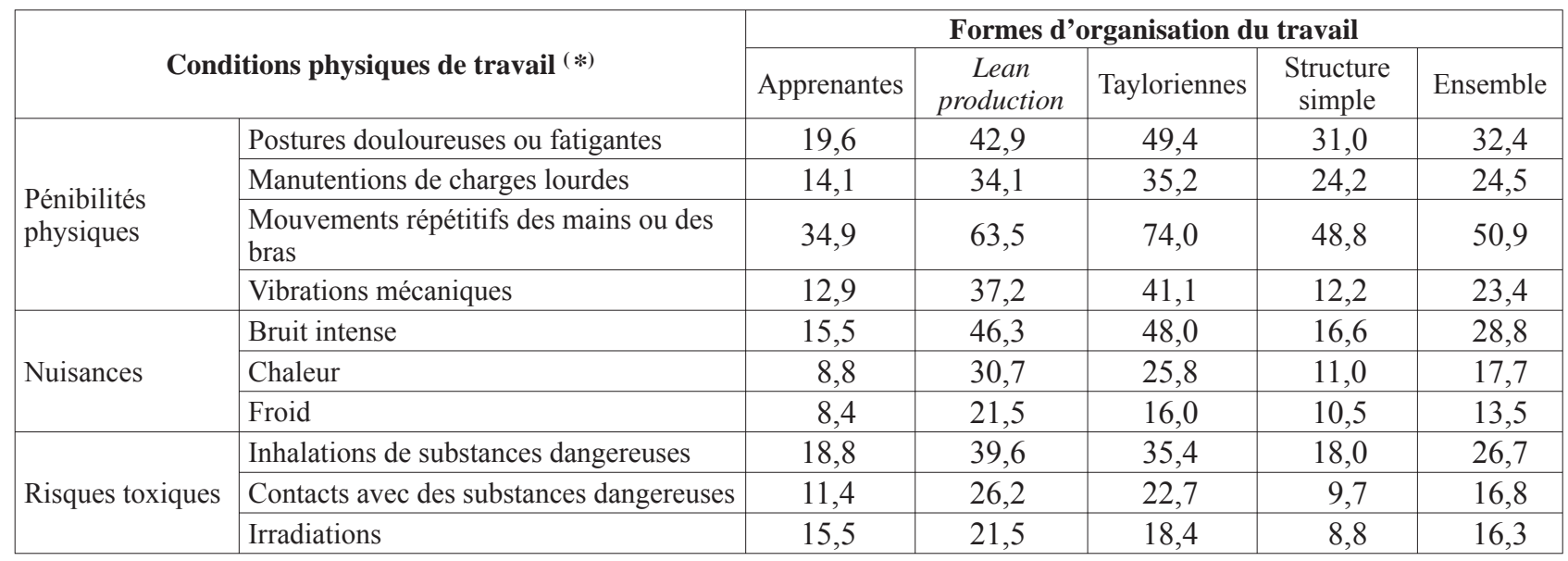

${ }^{(*)}$ : durées d'exposition: au moins la moitié du temps pour les pénibilités physiques et les nuisances; au moins le quart du temps pour les risques toxiques hors irradiations; au moins un instant pour les irradiations.

Lecture: 19,6\% des salariés des organisations apprenantes sont exposés à des postures douloureuses ou fatigantes au moins la moitié du temps de travail.

Champ: salariés des secteurs marchands, hors agriculture et services domestiques, travaillant dans des établissements d'au moins dix personnes.

Source: troisième enquête européenne sur les conditions de travail, Fondation européenne pour l'amélioration des conditions de vie et de travail.

Tableau 2

Conditions physiques de travail et formes d'organisation du travail (odds ratios de modèles logit avec contrôle structurel) (odds ratios)

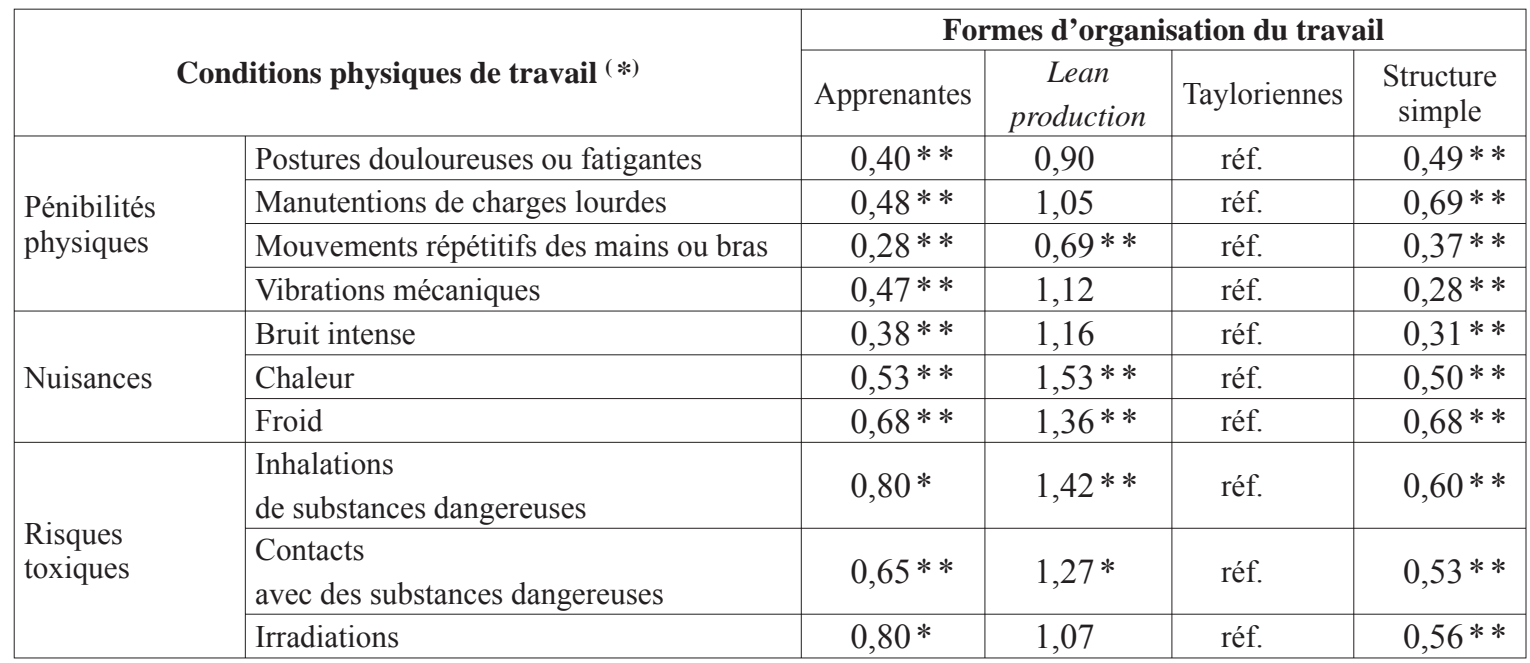

(*): durées d'exposition: au moins la moitié du temps pour les pénibilités physiques et les nuisances; au moins le quart du temps pour les risques toxiques hors irradiations; au moins un instant pour les irradiations.

Lecture: l'odds ratio 0,40 estime, toutes choses égales par ailleurs, le ratio entre le rapport des probabilités pour les salariés des organisations apprenantes d'être exposés à des postures douloureuses ou fatigantes et de ne pas l'être et ce rapport de probabilités pour les salariés des organisations tayloriennes (choisies comme référence). Inférieur à 1, cet odds ratio signifie qu'il est moins probable d'être exposé à des postures douloureuses ou fatigantes dans les organisations apprenantes que dans les organisations tayloriennes. Supérieur à 1 ou égal à 1, il traduirait une probabilité plus forte ou égale. La significativité statistique des odds ratios aux seuils $1 \%$ et $5 \%$ est indiquée par** et*.

Variables de contrôle structurel du modèle logit (non représentées dans le tableau): secteur, taille des établissements, groupe socioprofessionnel, pays, type de contrat de travail, genre, âge et ancienneté.

Champ: salariés des secteurs marchands, hors agriculture et services domestiques, travaillant dans des établissements d'au moins dix personnes. Source: troisième enquête européenne sur les conditions de travail, Fondation européenne pour l'amélioration des conditions de vie et de travail 
dans le tableau 2(7). Ainsi, toutes choses égales par ailleurs, par rapport à la situation de référence des salariés des organisations tayloriennes, la probabilité d'être exposé à des conditions physiques de travail pénibles ou risquées est significativement beaucoup plus faible dans les organisations apprenantes ou de structure simple. À l'inverse, elle est dans presque tous les cas plus élevée dans les organisations en lean production et ce, de façon statistiquement significative pour les nuisances thermiques et les risques d'inhalations ou de contacts dangereux. Seule l'exposition à des mouvements répétitifs des mains ou des bras s'y avère significativement moins probable que dans les organisations tayloriennes. Les résultats de l'analyse logistique convergent donc pleinement avec ceux de l'analyse descriptive. Par ailleurs, ils apportent quelques nuances en montrant que les expositions à des postures douloureuses ou fatigantes ou à des vibrations mécaniques ne sont pas significativement différentes pour les salariés des organisations tayloriennes et ceux des organisations en lean production.

\section{Conditions horaires de travail}

Les conditions horaires de travail sont étudiées selon trois dimensions: les durées de travail longues; les horaires atypiques; et les horaires flexibles. L'enquête européenne sur les conditions de travail permet de construire de multiples variables relatives à ces trois dimensions. Deux variables de durées de travail longues sont retenues: les durées hebdomadaires longues (au moins 45 heures par semaine); et les durées quotidiennes fréquemment longues (plus de 10 heures plus de cinq fois par mois (8)). Cinq variables d'horaires de travail atypiques sont étudiées: le travail de nuit (plus de cinq fois par mois); le travail en soirée (plus de dix fois par mois); le travail le dimanche (au moins une fois par mois); le travail le samedi (au moins une fois par mois); et le travail posté. Enfin la flexibilité des horaires de travail est analysée à partir de quatre variables: la flexibilité quotidienne des durées de travail (nombre d'heures de travail variable chaque jour); la flexibilité hebdomadaire du nombre de journées de travail (nombre de jours de travail variable chaque semaine); les horaires variables; et les changements mensuels d'horaires de travail (au moins une fois par mois).

(7) Les résultats complets des régressions logistiques des variables de conditions physiques de travail, incluant l'estimation des effets des variables de contrôle structurel, figurent dans VALEYRE (2006a). Il en est de même pour les résultats complets concernant les autres variables de conditions de travail étudiées dans cet article.

(8) Le choix d'une fréquence mensuelle des journées longues plus élevée se heurte à des problèmes de signification statistique. Il en est de même dans le choix des fréquences des horaires atypiques.
Les conditions horaires de travail présentent des disparités importantes entre formes d'organisation du travail, comme le montre le tableau 3 infra. La pratique d'un travail en horaires atypiques comme le travail posté ou effectué de nuit, en soirée ou le dimanche, est nettement plus développée dans les organisations en lean production et les organisations tayloriennes, et la moins répandue dans les organisations apprenantes. Le travail le samedi est moins contrasté, avec toutefois une légère prépondérance dans les organisations en lean production et un moindre niveau dans les organisations apprenantes. Les disparités organisationnelles des horaires atypiques présentent donc une grande similitude avec celles qui sont mises en évidence dans le cas des conditions physiques de travail. En revanche, la situation est bien différente en ce qui concerne les horaires longs ou flexibles. Les durées de travail longues et les changements d'horaires dans le mois sont les plus fréquents pour les salariés travaillant dans les organisations apprenantes et, dans une moindre mesure, dans les organisations en lean production, et sont les moins diffusés dans les organisations tayloriennes. Quant à la flexibilité horaire définie en termes d'horaires variables ou de nombre d'heures de travail variable chaque jour, elle est également la plus importante dans les organisations apprenantes et la moins développée dans les organisations tayloriennes, mais est relativement moins fréquente dans les organisations en lean production. Relevons par ailleurs que la flexibilité du nombre de jours de travail chaque semaine varie peu entre formes d'organisation du travail.

Les analyses de régression logistique avec contrôle structurel confirment en grande partie ces résultats ( $c f$. tableau 4 infra). Par rapport à la situation de référence des salariés des organisations tayloriennes, la probabilité d'avoir des horaires atypiques est significativement plus élevée pour les salariés des organisations en lean production (à l'exception du travail le soir) et plus faible pour ceux des organisations apprenantes ou de structure simple (à l'exception du travail le samedi). La probabilité d'être soumis à des durées de travail longues ou à des horaires flexibles est en général significativement plus forte à la fois dans les organisations apprenantes et dans les organisations en lean production, et uniquement dans les organisations en lean production dans le cas de la variabilité du nombre de jours de travail chaque semaine. Le contrôle de l'influence des variables structurelles à l'aide d'analyses logistiques introduit donc des différences mineures par rapport aux résultats de l'analyse descriptive. Elle fait notamment apparaître que le travail posté est nettement et significativement plus développé dans les organisations en lean production que dans les organisations tayloriennes, à l'inverse de ce que montrent les pourcentages du tableau 3 . 
Tableau 3

Conditions horaires de travail selon les formes d'organisation du travail ( \% de salariés exposés)

\begin{tabular}{|c|c|c|c|c|c|c|}
\hline \multirow{2}{*}{\multicolumn{2}{|c|}{ Conditions horaires de travail }} & \multicolumn{5}{|c|}{ Formes d'organisation du travail } \\
\hline & & Apprenantes & $\begin{array}{c}\text { Lean } \\
\text { production }\end{array}$ & Tayloriennes & $\begin{array}{l}\text { Structure } \\
\text { simple }\end{array}$ & Ensemble \\
\hline \multirow[b]{2}{*}{ Durées longues } & Au moins 45 heures par semaine & 19,5 & 16,9 & 9,8 & 11,7 & 16,0 \\
\hline & $\begin{array}{l}\text { Plus de } 10 \text { heures par jour ( }>5 \text { fois par } \\
\text { mois) }\end{array}$ & 16,0 & 14,7 & 8,0 & 9,3 & 13,3 \\
\hline \multirow{5}{*}{$\begin{array}{l}\text { Horaires } \\
\text { atypiques }\end{array}$} & $\begin{array}{l}\text { Travail } \\
\text { de nuit ( }>5 \text { fois par mois) }\end{array}$ & 6,6 & 20,4 & 16,1 & 11,2 & 12,6 \\
\hline & Travail le soir ( $>10$ fois par mois) & 11,0 & 16,2 & 16,2 & 14,7 & 13,9 \\
\hline & $\begin{array}{l}\text { Travail } \\
\text { le dimanche }\end{array}$ & 17,6 & 28,0 & 21,4 & 19,8 & 21,5 \\
\hline & $\begin{array}{l}\text { Travail } \\
\text { le samedi }\end{array}$ & 41,9 & 50,5 & 44,3 & 45,8 & 45,4 \\
\hline & Travail posté & 12,7 & 37,8 & 39,3 & 22,0 & 25,2 \\
\hline \multirow{4}{*}{$\begin{array}{l}\text { Horaires } \\
\text { flexibles }\end{array}$} & Nombre d'heures variable chaque jour & 42,0 & 31,4 & 20,0 & 35,1 & 34,7 \\
\hline & $\begin{array}{l}\text { Nombre } \\
\text { de jours variable chaque semaine }\end{array}$ & 21,2 & 23,4 & 19,5 & 22,1 & 21,7 \\
\hline & Horaires variables & 36,8 & 25,6 & 16,5 & 28,3 & 29,2 \\
\hline & Changements d'horaires dans le mois & 28,5 & 24,6 & 21,5 & 22,4 & 25,3 \\
\hline
\end{tabular}

Lecture: 19,5\% des salariés des organisations apprenantes travaillent au moins 45 heures par semaine.

Champ: salariés des secteurs marchands, hors agriculture et services domestiques, travaillant dans des établissements d'au moins dix personnes.

Source: troisième enquête européenne sur les conditions de travail, Fondation européenne pour l'amélioration des conditions de vie et de travail.

Tableau 4

\section{Conditions horaires de travail et formes d'organisation du travail (odds ratios de modèles logit avec contrôle structurel)}

\begin{tabular}{|c|c|c|c|c|c|}
\hline \multirow{2}{*}{\multicolumn{2}{|c|}{$\begin{array}{l}\text { Conditions horaires } \\
\text { de travail }\end{array}$}} & \multicolumn{4}{|c|}{ Formes d'organisation du travail } \\
\hline & & \multirow{2}{*}{$\begin{array}{c}\text { Apprenantes } \\
1,51 * *\end{array}$} & \multirow{2}{*}{$\begin{array}{c}\begin{array}{c}\text { Lean } \\
\text { production }\end{array} \\
1,36^{*}\end{array}$} & \multirow{2}{*}{$\begin{array}{c}\text { Tayloriennes } \\
\text { réf. }\end{array}$} & \multirow{2}{*}{$\begin{array}{c}\begin{array}{c}\text { Structure } \\
\text { simple }\end{array} \\
1,06\end{array}$} \\
\hline \multirow[b]{2}{*}{ Durées longues } & Au moins 45 heures par semaine & & & & \\
\hline & $\begin{array}{l}\text { Plus de } 10 \text { heures par jour ( }>5 \text { fois par } \\
\text { mois) }\end{array}$ & $1,56 * *$ & $1,42 * *$ & réf. & 0,98 \\
\hline \multirow{5}{*}{ Horaires atypiques } & Travail de nuit (> 5 fois par mois) & $0,51 * *$ & $1,31 *$ & réf. & $0,68 * *$ \\
\hline & Travail le soir (> 10 fois par mois) & $0,69 * *$ & 0,93 & réf. & $0,71 * *$ \\
\hline & $\begin{array}{l}\text { Travail } \\
\text { le dimanche }\end{array}$ & $0,70 * *$ & $1,25 *$ & réf. & $0,70 * *$ \\
\hline & $\begin{array}{l}\text { Travail } \\
\text { le samedi }\end{array}$ & 1,07 & $1,36 * *$ & réf. & 0,89 \\
\hline & Travail posté & $0,46 * *$ & $1,21 *$ & réf. & $0,53 * *$ \\
\hline \multirow{4}{*}{ Horaires flexibles } & Nombre d'heures variable chaque jour & $1,77 * *$ & $1,54 * *$ & réf. & $1,59 * *$ \\
\hline & $\begin{array}{l}\text { Nombre de jours variable chaque } \\
\text { semaine }\end{array}$ & 1,05 & $1,32 * *$ & réf. & 0,94 \\
\hline & Horaires variables & $1,69 * *$ & $1,38 * *$ & réf. & $1,40 * *$ \\
\hline & Changements d'horaires dans le mois & $1,49 * *$ & $1,25 *$ & réf. & 0,95 \\
\hline
\end{tabular}

Lecture: l'odds ratio 1,51 estime, toutes choses égales par ailleurs, le ratio entre le rapport des probabilités pour les salariés des organisations apprenantes de travailler au moins 45 heures par semaine et de travailler moins de 45 heures et ce rapport de probabilités pour les salariés des organisations tayloriennes (choisies comme référence). Supérieur à 1 , cet odds ratio signifie qu'il est plus probable de travailler au moins 45 heures par semaine dans les organisations apprenantes que dans les organisations tayloriennes. Inférieur à 1 ou égal à 1, il traduirait une probabilité plus faible ou égale. La significativité statistique des odds ratios aux seuils $1 \%$ et $5 \%$ est indiquée par** et*.

Variables de contrôle structurel du modèle logit (non représentées dans le tableau): secteur, taille des établissements, groupe socioprofessionnel, pays, type de contrat de travail, genre, âge et ancienneté.

Champ: salariés des secteurs marchands, hors agriculture et services domestiques, travaillant dans des établissements d'au moins dix personnes.

Source: troisième enquête européenne sur les conditions de travail, Fondation européenne pour l'amélioration des conditions de vie et de travail. 
Intensité du travail

Les dimensions horaires du travail qui viennent d'être étudiées ne couvrent pas l'ensemble des dimensions temporelles des conditions de travail. Il convient aussi d'examiner les pressions temporelles que subissent les salariés dans le cadre de leurs horaires de travail, notamment en termes d'intensité du travail. Trois variables d'intensité du travail sont construites sur la base de l'enquête européenne sur les conditions de travail: la soumission quasi permanente (tout le temps ou presque) à des cadences de travail élevées; la soumission quasi permanente à des délais très stricts et très serrés; et le manque de temps pour terminer le travail.

Comme les variables d'intensité du travail ressentie par les salariés sont liées aux variables de contraintes de rythme de travail qui s'exercent sur eux (Boisard et al., 2002; VAleyre, 2004) et que ces dernières interviennent dans la différenciation des classes de formes d'organisation du travail, il n'est pas surprenant d'observer d'importantes différences d'intensité du travail entre organisations apprenantes ou de structure simple d'une part et organisations tayloriennes ou en lean production d'autre part. Comme on peut le constater dans le tableau 5 infra, les salariés qui estiment être soumis tout le temps ou presque à des cadences élevées ou à des délais très stricts et très serrés, sont beaucoup plus nombreux dans les organisations en lean production et les organisations tayloriennes que dans les organisations apprenantes et les organisations de structure simple. La pression des délais s'avère particulièrement élevée dans les organisations en lean production. Par ailleurs, il apparaît que la situation est beaucoup moins contrastée en ce qui concerne le manque de temps pour terminer le travail. Elle est comparable dans les organisations apprenantes, en lean production ou tayloriennes, et légèrement plus favorable dans les organisations de structure simple.

Tableau 5

Intensité du travail selon les formes d'organisation du travail ( \% de salariés soumis à un travail intense)

\begin{tabular}{|l|c|c|c|c|c|}
\hline \multirow{2}{*}{} & \multicolumn{5}{|c|}{ Formes d'organisation du travail } \\
\cline { 2 - 6 } & Apprenantes & $\begin{array}{c}\text { Lean } \\
\text { production }\end{array}$ & Tayloriennes & $\begin{array}{c}\text { Structure } \\
\text { simple }\end{array}$ & Ensemble \\
\hline Cadences élevées tout le temps ou presque & 20,5 & 39,1 & 39,3 & 21,9 & 28,6 \\
\hline Délais serrés tout le temps ou presque & 31,0 & 50,8 & 39,0 & 22,5 & 36,0 \\
\hline Manque de temps pour terminer le travail & 24,0 & 25,4 & 23,8 & 18,2 & 23,3 \\
\hline
\end{tabular}

Lecture : 20,5\% des salariés des organisations apprenantes sont soumis à des cadences élevées tout le temps ou presque.

Champ: salariés des secteurs marchands, hors agriculture et services domestiques, travaillant dans des établissements d'au moins dix personnes.

Source: troisième enquête européenne sur les conditions de travail, Fondation européenne pour l'amélioration des conditions de vie et de travail.

Tableau 6

Intensité du travail et formes d'organisation du travail (odds ratios de modèles logit avec contrôle structurel) (odds ratios)

\begin{tabular}{|l|c|c|c|c|}
\hline \multirow{2}{*}{} & \multicolumn{3}{|c|}{ Formes d'organisation du travail } \\
\cline { 2 - 5 } & Apprenantes & $\begin{array}{c}\text { Lean } \\
\text { production }\end{array}$ & $\begin{array}{c}\text { Tayloriennes } \\
\text { Structure } \\
\text { simple }\end{array}$ \\
\hline Cadences élevées tout le temps ou presque & $0,48 * *$ & 1,12 & réf. & $0,44 * *$ \\
\hline Délais serrés tout le temps ou presque & $0,58 * *$ & $1,36 * *$ & réf. & $0,43 * *$ \\
\hline Manque de temps pour terminer le travail & $0,79 *$ & 1,00 & réf. & $0,67 * *$ \\
\hline
\end{tabular}

Lecture: l'odds ratio 0,48 estime, toutes choses égales par ailleurs, le ratio entre le rapport des probabilités pour les salariés des organisations apprenantes d'être soumis à des cadences élevées tout le temps ou presque et de ne pas l'être et ce rapport de probabilités pour les salariés des organisations tayloriennes (choisies comme référence). Inférieur à 1, cet odds ratio signifie qu'il est moins probable d'être soumis à des cadences élevées tout le temps ou presque dans les organisations apprenantes que dans les organisations tayloriennes. Supérieur à 1 ou égal à 1 , il traduirait une probabilité plus forte ou égale. La significativité statistique des odds ratios aux seuils $1 \%$ et $5 \%$ est indiquée par** et*.

Variables de contrôle structurel du modèle logit (non représentées dans le tableau): secteur, taille des établissements, groupe socioprofessionnel, pays, type de contrat de travail, genre, âge et ancienneté.

Champ: salariés des secteurs marchands, hors agriculture et services domestiques, travaillant dans des établissements d'au moins dix personnes.

Source: troisième enquête européenne sur les conditions de travail, Fondation européenne pour l'amélioration des conditions de vie et de travail. 


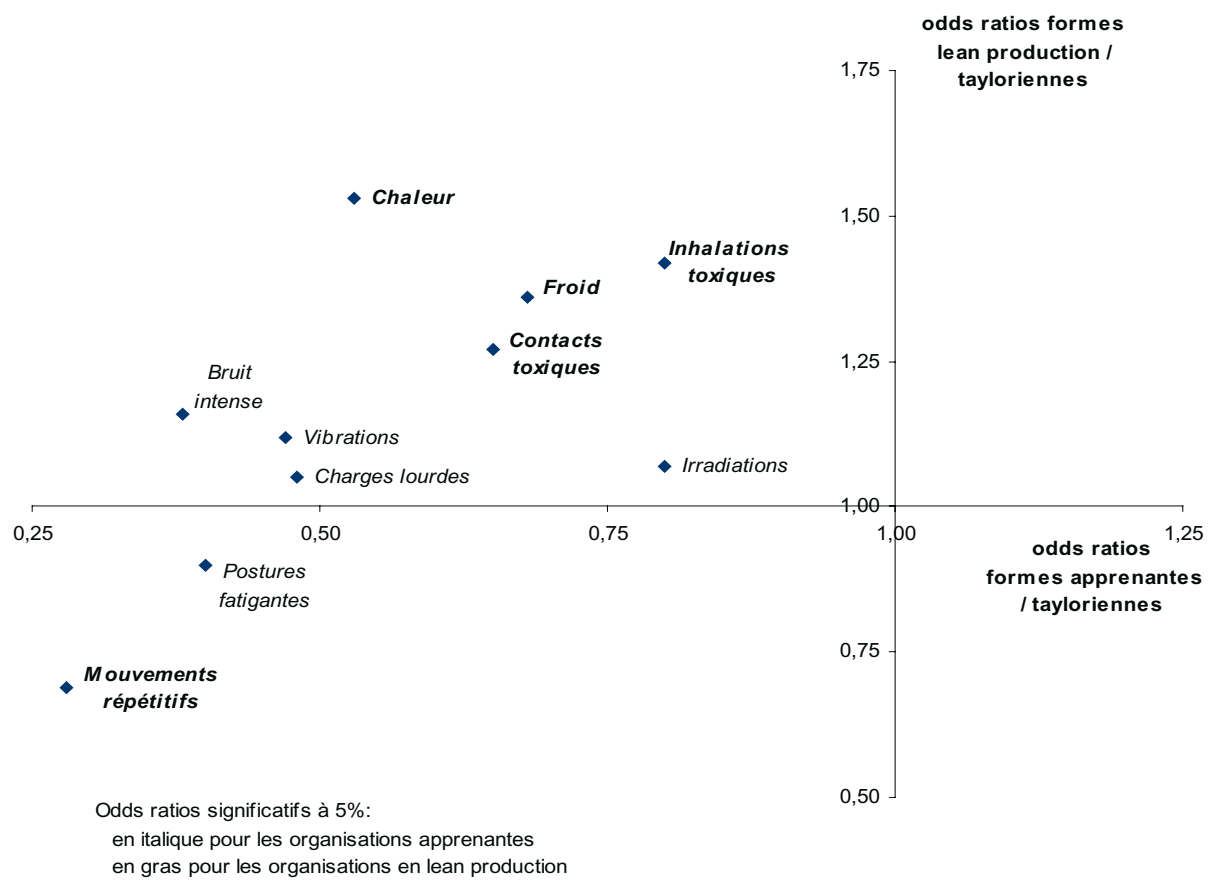

Lecture: les coordonnées du graphique représentent les odds ratios d'exposition des salariés aux diverses conditions physiques de travail selon qu'ils travaillent dans des organisations apprenantes (en abscisse) ou en lean production (en ordonnée), en référence à la situation des salariés des organisations tayloriennes. Ainsi, par rapport aux salariés des organisations tayloriennes, l'exposition à la chaleur est moins probable pour les salariés des organisations apprenantes et plus probable pour ceux des organisations en lean production. Les odds ratios de ce graphique sont issus du tableau 2.

Ces résultats sont confortés dans une large mesure par les régressions logistiques avec contrôle structurel ( $c f$. tableau 6). En référence à la situation des salariés des organisations tayloriennes, la probabilité d'être soumis à une forte intensité du travail, qu'elle soit liée à des cadences de travail élevées, à des délais serrés ou au manque de temps, est significativement bien moindre dans les organisations apprenantes ou de structure simple. En revanche, elle est au moins égale dans les organisations en lean production, voire significativement plus élevée dans le cas de la pression des délais serrés. L'analyse toutes choses égales par ailleurs conduit ainsi à un résultat légèrement différent en ce qui concerne le manque de temps pour terminer le travail qui apparaît significativement moindre dans les organisations apprenantes que dans les organisations tayloriennes, alors que l'analyse descriptive le situe à un niveau très proche.

\section{Des conditions de travail contrastées dans les organisations innovantes}

Les résultats de la recherche confirment les hypothèses formulées en introduction sur les conditions de travail dans les nouvelles formes d'organisation, apprenantes ou en lean production. De nombreuses caractéristiques des conditions de travail relatives notamment aux pénibilités physiques, aux nuisances sonores et thermiques, aux risques toxiques, aux horaires atypiques et à l'intensité du travail, sont beaucoup plus favorables pour les salariés des organisations apprenantes que pour ceux des organisations en lean production. Seuls les durées de travail longues ou les horaires flexibles sont plus fréquents dans les organisations apprenantes. La comparaison des conditions de travail des salariés exerçant leur activité dans de nouvelles formes d'organisation avec celles que l'on observe dans les organisations tayloriennes fait apparaître des situations très contrastées entre organisations apprenantes et organisations en lean production, comme l'illustrent les figures 1 et 2 . Les conditions de travail des salariés des organisations apprenantes sont en général bien meilleures que celles des organisations tayloriennes, les horaires longs ou flexibles faisant toutefois exception. En revanche, ce n'est pas le cas pour les salariés des organisations en lean production. En effet, ces derniers sont exposés à des conditions de travail souvent moins bonnes que dans les organisations tayloriennes, notamment en matière de nuisances thermiques, de risques toxiques, d'horaires atypi- 
Figure 2

Conditions d'horaires de travail dans les nouvelles formes d'organisation

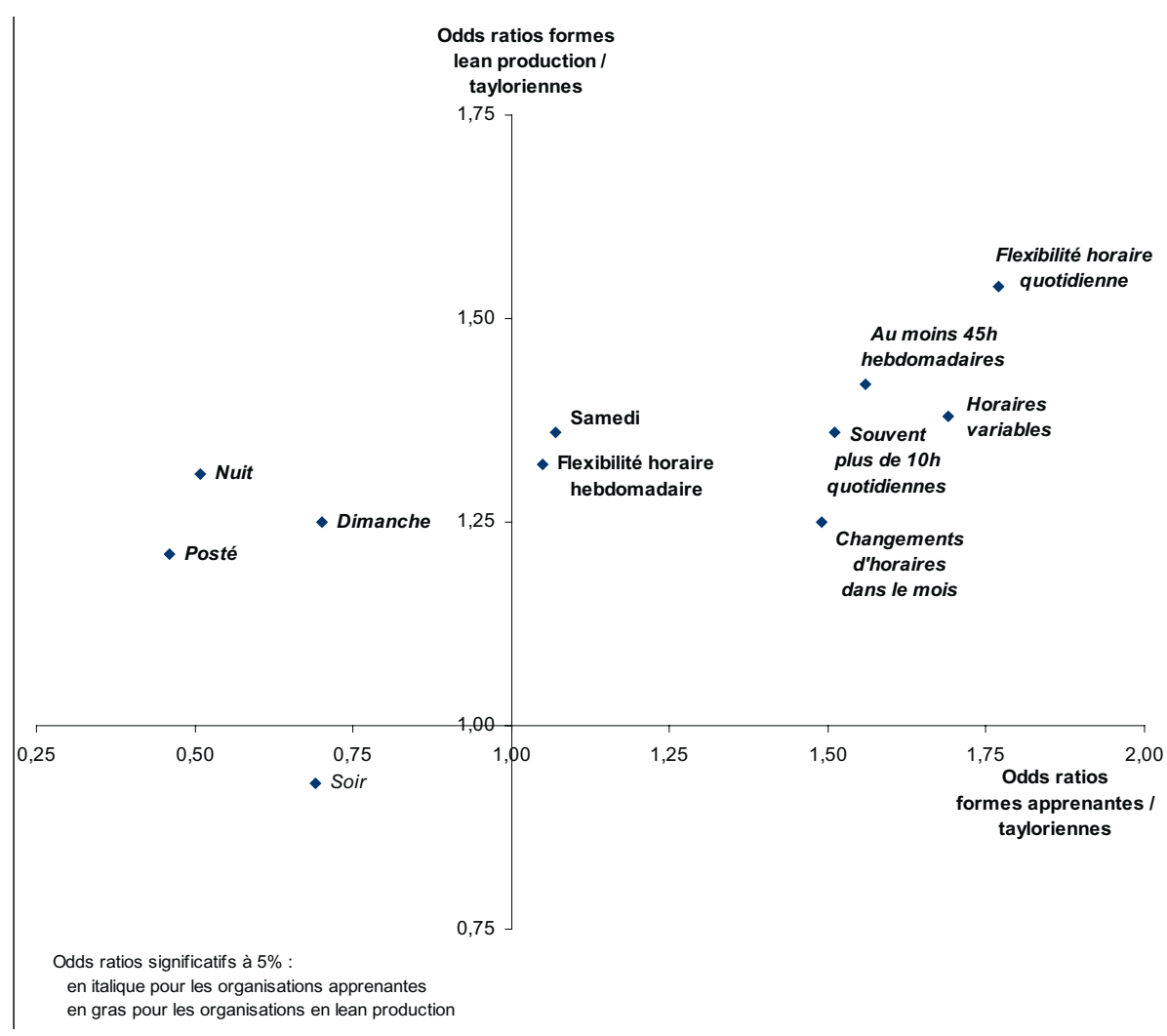

Lecture: les coordonnées du graphique représentent les odds ratios de soumission des salariés aux diverses conditions horaires de travail selon qu'ils travaillent dans des organisations apprenantes (en abscisse) ou en lean production (en ordonnée), en référence à la situation des salariés des organisations tayloriennes. Ainsi, par rapport aux salariés des organisations tayloriennes, l'exercice d'un travail de nuit est moins probable pour les salariés des organisations apprenantes et plus probable pour ceux des organisations en lean production. Les odds ratios de ce graphique sont issus du tableau 4.

ques, longs ou flexibles, et d'intensité du travail liée aux délais, ou comparables en ce qui concerne un grand nombre de pénibilités physiques, les nuisances sonores, les irradiations, le travail en soirée ou l'intensité du travail liée à des cadences élevées ou au manque de temps. Seule la moindre exposition à des mouvements répétitifs des mains ou des bras fait exception en plaçant les salariés des organisations en lean production en situation plus favorable. Ainsi, l'obtention de conditions de travail meilleures dans les nouvelles formes d'organisation du travail que dans les organisations tayloriennes ne se vérifie que partiellement, clairement dans les organisations apprenantes, mais pas dans les organisations en lean production. La distinction entre formes nouvelles d'organisation du travail permet donc de circonscrire le paradoxe d'innovations organisationnelles ne contribuant pas à l'amélioration des conditions de travail, en montrant qu'il ne vaut que pour les organisations en lean production.
Dans les années 1970, les innovations organisationnelles visant à dépasser les limites économiques et sociales du modèle taylorien/fordien ont été conçues dans une double perspective d'amélioration des performances économiques et productives des entreprises et des conditions de travail de la maind'œuvre. Par la suite, la montée et l'ampleur des problèmes d'emploi ont rendu moins prioritaires les préoccupations de qualité de vie au travail. Les innovations organisationnelles qui se sont diffusées depuis la fin des années 1980 ne s'accompagnent plus nécessairement d'une amélioration des conditions de travail, notamment lorsqu'elles relèvent des principes de la lean production et de la logique d'intensification du travail dont elle est porteuse. En outre, certaines entreprises ont adopté des stratégies d'évitement des problèmes de conditions de travail, en automatisant ou en informatisant la production, en recourant à une main-d'œuvre immigrée ou 
précaire peu encline à rejeter de mauvaises conditions de travail au risque de perdre son emploi, et en externalisant ou en délocalisant certaines activités (Ben Aissa, 2005).

En conséquence, la question de l'amélioration des conditions de travail n'a rien perdu de son actualité et de son urgence (Bressol, 2004). Pour surmonter le paradoxe d'organisations innovantes ne contribuant pas à une meilleure qualité de vie au travail, AskeNAZY (2004) invite à prendre en exemple le «miracle» américain. En effet, depuis une dizaine d'années, on observe une diminution importante des accidents du travail aux États-Unis. Cette inflexion de tendance que vient favoriser un contexte de pleinemploi, est la résultante de l'action des pouvoirs publics, notamment par l'intermédiaire de l'Office of Safety and Health Administration (OSHA), des organisations syndicales, des compagnies d'assurance et des entreprises confrontées à des coûts devenus considérables en matière d'accidents du travail. Elle est, de plus, renforcée par la transparence et la large diffusion des informations sur la sécurité au travail qu'amplifie la généralisation de l'accès à l'Internet. Il conviendrait cependant d'examiner dans quelle mesure les évolutions constatées pour les accidents du travail s'étendent à l'ensemble des conditions de travail. Compte tenu des principes qui les fondent, il est peu probable que les formes d'organisation du travail en lean production soient en mesure d'intégrer pleinement les préoccupations d'amélioration des conditions de travail. Ce serait probablement les faire changer de nature. Sans attendre des réponses à ces interrogations, les résultats présentés dans cet article invitent, quant à eux, à rechercher des solutions dans les pratiques innovantes des organisations du travail apprenantes qui se sont largement diffusées en Europe, tout particulièrement dans les pays scandinaves, en prenant en compte la qualité de vie au travail dans la continuité des principes du modèle sociotechnique.

\section{Bibliographie}

Appay B. (1993), «Individu ou collectif: question à la sociologie du travail et des professions. L'autonomie contrôlée», Cahiers du GEDISST, nº 6, pp. 57-92.

Appelbaum E., Batt R. (1994), The new American Workplace, Ithaca, New York, ILR Press.

Arnaudo B., Magaud-Camus I., Sandret N., Coutrot T., Floury M.-C., Guignon N., Hamon-Cholet S., WALtisperger D. (2004), «L'exposition aux risques et aux pénibilités du travail de 1994 à $2003 »$, Premières Synthèses, $\mathrm{n}^{0}$ 52.1, DARES, décembre.

AskenAzy Ph. (2002), La croissance moderne. Organisations innovantes du travail, Paris, Economica.

Askenazy Ph. (2004), Les désordres du travail, Paris, Seuil et République des Idées.

Askenazy Ph., Caroli E. (2003), New Organizational Practices and Well-Being at Work: Evidence for France in 1998, LEA Working Paper 03-11.

Ben Aissa H. (2005), Histoire des conditions de travail dans le monde industriel en France: 1848-2000, Paris, L'Harmattan.

Boisard P., Cartron D., Gollac M., Valeyre A. (2002), Temps et travail: l'intensité du travail, Luxembourg, Office des publications officielles des Communautés européennes.

Boyer R., Durand J.-P. (1993), L'après fordisme, Paris, Syros.
Boyer R., Freyssenet M. (2000), Les modèles productifs, Paris, La Découverte.

Bressol E. (2004), Organisation du travail et nouveaux risques pour la santé des travailleurs, Rapport au Conseil économique et social, éditions des Journaux officiels, $\mathrm{n}^{\mathrm{o}} 10$.

Bué J., Coutrot T., Puech I. (coord.) (2004), Conditions de travail: les enseignements de vingt ans d'enquêtes, Toulouse, Octarès.

Bunel M., Dayan J.-L., Desage G., Perraudin C., VAleyre A. (2006), Formes d'organisation du travail et relations de travail, rapport pour la DARES, Noisy-leGrand, Centre d'études de l'emploi, novembre, 230 p.

Cartron D., Gollac M. (2006), «Fast-work et mal travail», in Ph. Askenazy, D. CArtron, F. DE Coninck, M. Gollac (coord.), Organisation et intensité du travail, Toulouse, Octarès, pp. 227-237.

Coutrot T. (1995) «Gestion de l'emploi et organisation du travail dans les entreprises innovantes», Travail et Emploi, no 64, avril, pp. 79-87.

Coutrot T. (1998), L'entreprise néolibérale, nouvelle utopie capitaliste?, Paris, La Découverte.

Coutrot T. (2000), «Innovation dans le travail: la pression de la concurrence internationale, l'atout des qualifications », Premières Synthèses, 2000.3, nº 09.02, MESDARES. 
Daubas-Letourneux V., Thébaud-Mony A. (2003), «Organisation du travail et santé dans l'Union européenne», Travail et Emploi, no 96, octobre, pp. 935.

DAvezies Ph. (1999), «Évolution des organisations du travail et atteintes à la santé», Travailler, no 3, pp. 7-22.

Durand J.-P., Stewart P., Castillo J.-J. (coord.) (1998), Teamwork in the Automobile Industry. Radical Change or Passing Fashion? London, MacMillan.

Edwards P., Geary J., Sisson K. (2004), «Les nouvelles formes d'organisation du travail: moyens de transformation, d'exploitation ou de contrôle?», in G. Murray, J. Bélanger, A. Giles, P.-A. Lapointe (sous la direction de), L'organisation de la production et du travail: vers un nouveau modèle? Laval, Canada, Presses de l'université de Laval, pp. 63-117.

Emery F., Trist E. L. (1960), «Socio-technical systems, in C. W. Churchman, M. Verhulst (eds), Management Science. Models and Techniques, vol. 2, London, Pergamon Press.

Fröhlich D., Pekruhl U. (1996), Direct Participation and Organisational Change. Fashionable but Misunderstood? Luxembourg, Office for official publications of the European communities.

Gollac M. (1995), Donner un sens aux données: l'exemple des enquêtes statistiques sur les conditions de travail, Dossier du CEE, $n^{\circ} 3$, Noisy-le-Grand, Centre d'études de l'emploi.

Gollac M., Volkoff (1996), «Citius, altius, fortius. L'intensification du travail», Actes de la Recherche en Sciences Sociales, no 114, septembre, pp. 54-67.

Gollac M., Volkoff S. (2000), Les conditions de travail, Paris, La Découverte.

Greenan N., Hamon-Cholet S. (2000), «Les salariés industriels face aux changements organisationnels", Premières Synthèses, 2000.03, nº 09.3, MES-DARES.

Hamon-Cholet S. (2004), «La charge mentale: fardeau ou aiguillon?», in BuÉ J., Coutrot T., Puech I. (coord.), Conditions de travail: les enseignements de vingt ans d'enquêtes, Toulouse, Octarès, pp. 89-100.

Linhart D. (1994), La modernisation des entreprises, Paris, La Découverte.

LoRENZ E., VALEYRE A. (2004), Les formes d'organisation du travail dans les pays de l'Union européenne, Document de travail, $n^{\circ} 32$, Noisy-le-Grand, Centre d'études de l'emploi, juin, $32 \mathrm{p}$.
Lorenz E., VAleyre A. (2005a), «Les formes d'organisation du travail dans les pays de l'Union européenne», Travail et Emploi, no 102, avril, pp.91105.

LOREnZ E., VALeyre A. (2005b), “Organisational Innovation, Human Resource Management and Labour Market Structure: a Comparison of the EU-15", The Journal of Industrial Relations, Vol. 47, December, pp. 424-442.

MACDuffie J.P., KRAFCIK J. (1992), “Integrating Technology and Human Resources for High Performance Manufacturing: Evidence From the International Auto Industry", in T. Kochan, M. Useem (eds), Transforming Organisations, New York, Oxford University Press, pp. 209-226.

Merllié D., Paoli P. (2001), Third European Survey on Working Conditions (2000), Luxembourg, Office for official publications of the European communities.

Mintzberg H. (1982), Structure et dynamique des organisations, Paris, Éditions d'Organisation.

Moati P., Pouquet L. (2002), «Les nouvelles pratiques organisationnelles révèlent-elles l'émergence d'un nouveau modèle productif?», Économie appliquée, tome LV, no 3, pp. 63-90.

Osterman P. (1994), "How Common Is Workplace Transformation and Who Adopts It?", Industrial and Labor Relations Review, vol. 47, n 2, pp. 173-188.

Osterman P. (2000), "Work Reorganization in an Era of Restructuring: Trends in Diffusion and Effects on Employee Welfare", Industrial and Labor Relations Review, vol. 53, pp. 179-196.

VAleyre A. (2004), «Les nouvelles formes d'intensification du travail industriel: logiques technologiques, organisationnelles et économiques», Économies et Sociétés, série «Socio-Économie du travail», AB, nº 24, novembre, pp. 1993-2027.

Valeyre A. (2006a), Organisations du travail, conditions de travail et santé au travail dans l'Union européenne, rapport pour la DARES, Noisy-le-Grand, Centre d'études de l'emploi, mai, $97 \mathrm{p}$.

Valeyre A. (2006b), Conditions de travail et santé au travail dans l'Union européenne: des situations contrastées selon les formes d'organisation, Document de travail, $\mathrm{n}^{\mathrm{o}} 73$, Noisy-le-Grand, Centre d'études de l'emploi, novembre, $48 \mathrm{p}$.

Womack J.P., Jones D.T., Roos D. (1990), The Machine that changed the World, New York, Rawson Associates. 